\title{
On the set of zeros of bicomplex polynomials
}

\author{
A. A. POGORUI and R. M. RODRÍGUEZ-DAGNINO* \\ Monterrey Institute of Technology, Monterrey, NL, México
}

Communicated by F. Sommen

(Received in final form 26 October 2005)

\begin{abstract}
In this article we study the roots or zeros of polynomials with bicomplex coefficients. Bicomplex algebra is a two-dimensional Clifford algebra over $\mathbb{C}$ with commutative multiplication, and is a generalization of complex numbers. We present a technique for computing zeros of bicomplex polynomials, and illustrate our main results with some examples.
\end{abstract}

Keywords: Bicomplex numbers; Polynomials; Zeros

Mathematics Subject Classifications: 30C15; 12D10

\section{Introduction}

Let $B$ be the bicomplex algebra, i.e., $B=\left\{a_{0}+i a_{1}+j a_{2}+e a_{3} \mid a_{i} \in \mathbb{R}\right\}$, where $i^{2}=$ $j^{2}=-e^{2}=-1$, and $i j=j i=e$, $i e=e i=-j, j e=e j=-i$. Bicomplex numbers seem to be introduced by Segre [1] for the first time in 1892, and they are also called tetranumbers [2], or quadriplex numbers in [3]. Many of their properties and relationships with hypercomplex functions have been recently studied in [4].

Many properties of roots of quaternionic polynomials have been presented in [5], however it is well-known that quaternionic algebra is not commutative, which makes their application more difficult to a variety of important problems. In spite of knowing that bicomplex algebra is commutative, the problem of finding the roots of bicomplex polynomials has not yet been approached in the literature. In this article, by using four lemmas we reduce the calculation of zeros of bicomplex polynomials to the calculation of zeros of complex polynomials. We present four detailed examples of second-, third-, and fourth-order equations to illustrate our main results.

*Corresponding author. Email: rmrodrig@itesm.mx 


\section{Some basic lemmas}

Lemma 2.1 The algebra $B$ has exactly two non-trivial idempotents $e_{+}=(1+e) / 2$ and $e_{-}=(1-e) / 2$. These idempotents possess the following properties:

(i) $e_{+}+e_{-}=1$;

(ii) $e_{+} e_{-}=0$.

Proof Suppose $e_{+}=(1+e) / 2, \quad e_{-}=(1-e) / 2, \quad$ then $\quad e_{+}^{2}=\left(1+2 e+e^{2}\right) / 4=$ $(1+e) / 2=e_{+}$. Similarly, $e_{-}^{2}=\left(1-2 e+e^{2}\right) / 4=(1-e) / 2=e_{-}$, i.e., $e_{+}$and $e_{-}$are idempotents of $B$. It is easy to see that there are no other idempotents.

The equalities $e_{+}+e_{-}=1$ and $e_{+} e_{-}=0$ are obvious.

Now, consider the principal ideals $I\left(e_{+}\right)$and $I\left(e_{-}\right)$that are generated by $e_{+}$and $e_{-}$, i.e., $I\left(e_{+}\right)=\left\{a e_{+} \mid a \in B\right\}$ and $I\left(e_{-}\right)=\left\{a e_{-} \mid a \in B\right\}$.

Lemma 2.2 For all $a \in I\left(e_{+}\right)$and for all $b \in I\left(e_{-}\right)$we have $a b=0$.

Proof The result follows directly by using $e_{+} e_{-}=0$ and the commutativity of $B$.

Lemma 2.3 The algebra $B$ can be decomposed in the direct sum: $I\left(e_{+}\right) \oplus I\left(e_{-}\right)$.

Proof We have $b=b\left(e_{+}+e_{-}\right)=b e_{+}+b e_{-}$for all $b \in B$, hence $B=I\left(e_{+}\right)+I\left(e_{-}\right)$. We shall show that $I\left(e_{+}\right) \cap I\left(e_{-}\right)=\{0\}$. Suppose $u \in I\left(e_{+}\right) \cap I\left(e_{-}\right)$. Then there exists $a \in I\left(e_{+}\right)$and $b \in I\left(e_{-}\right)$such that $u=a e_{+}=b e_{-}$. It remains to verify that this equality is possible only if $a=b=0$.

Suppose $a=a_{0}+i a_{1}+j a_{2}+e a_{3}$ and $b=b_{0}+i b_{1}+j b_{2}+e b_{3}$ are arbitrary bicomplex numbers. Substituting these numbers into $a e_{+}=b e_{-}$, we obtain

$$
\begin{aligned}
& a_{0}+a_{3}+i\left(a_{1}-a_{2}\right)+j\left(a_{2}-a_{1}\right)+e\left(a_{0}+a_{3}\right) \\
& \quad=b_{0}-b_{3}+i\left(b_{1}+b_{2}\right)+j\left(b_{1}+b_{2}\right)+e\left(b_{3}-b_{0}\right) .
\end{aligned}
$$

Equating the coefficients of the same imaginary units we conclude that $a=b=0$.

Lemma 2.4 Assume that $p \in I\left(e_{+}\right)$(resp. $p \in I\left(e_{-}\right)$), then there exists a number $c$ that can be represented as $c=c_{0}+i c_{1} ; c_{0}, c_{1} \in \mathbb{R}$ such that $p=c e_{+}\left(\right.$resp. $\left.p=c e_{-}\right)$.

Proof Suppose $p \in I\left(e_{+}\right)$, then there exists an $a=a_{0}+i a_{1}+j a_{2}+e a_{3}$ such that $p=a e_{+}, \quad$ i.e., $\quad p=\left(a_{0}+i a_{1}+j a_{2}+e a_{3}\right) e_{+}=(1 / 2)\left(a_{0}+a_{3}+i\left(a_{1}-a_{2}\right)+j\left(a_{2}-a_{1}\right)+\right.$ $\left.e\left(a_{0}+a_{3}\right)\right)=\left(a_{0}+a_{3}+i\left(a_{1}-a_{2}\right)\right) e_{+}$. This proof is similar for $p \in I\left(e_{-}\right)$.

Remark 1 In the rest of the article we shall consider bicomplex numbers $c=c_{0}+i c_{1}$; $c_{0}, c_{1} \in \mathbb{R}$ as complex numbers.

\section{Main result}

Let $p_{n}(w)=a_{n} w^{n}+a_{n-1} w^{n-1}+\cdots+a_{1} w+a_{0}$ be a polynomial in $B$. Our purpose is to investigate the structure of the set of zeros of the equation

$$
p_{n}(w)=0
$$


We have from Lemma 2.3 that $w=w_{+}+w_{-}, a_{i}=a_{i}^{+}+a_{i}^{-}$, where $w_{+}, a_{i}^{+} \in I\left(e_{+}\right)$ and $w_{-}, a_{i}^{-} \in I\left(e_{-}\right)$.

It follows from Lemmas 2.2 and 2.3 that equation (1) can be reduced to the system:

$$
\left\{\begin{array}{l}
a_{n}^{+} w_{+}^{n}+a_{n-1}^{+} w_{+}^{n-1}+\cdots+a_{1}^{+} w_{+}+a_{0}^{+}=0 \\
a_{n}^{-} w_{-}^{n}+a_{n-1}^{-} w_{-}^{n-1}+\cdots+a_{1}^{-} w_{-}+a_{0}^{-}=0
\end{array}\right.
$$

Suppose $\mathcal{S}_{1}$ (resp. $\mathcal{S}_{2}$ ) is the set of zeros of the first (resp. the second) equation of the system (2). Then, the set of zeros $\mathcal{S}$ of equation (1) can be represented as $\mathcal{S}=\mathcal{S}_{1} \oplus \mathcal{S}_{2}$

Let us consider the following cases:

(i) There exists $i, j \geq 1$ such that $a_{i}^{+} \neq 0$ and $a_{j}^{-} \neq 0$. Denote by $a_{k}^{+}$and $a_{\ell}^{-}$non-zero coefficients which have maximal index $k \geq 1$ and $\ell \geq 1$ respectively. By using Lemma 2.4, system (2) can be reduced to the form:

$$
\left\{\begin{array}{l}
\left(c_{k} z^{k}+c_{k-1} z^{k-1}+\cdots+c_{1} z+c_{0}\right) e_{+}=0 \\
\left(c_{\ell} z^{\ell}+c_{\ell-1} z^{\ell-1}+\cdots+c_{1} z+c_{0}\right) e_{-}=0
\end{array}\right.
$$

where $\quad p_{k}(z)=c_{k} z^{k}+c_{k-1} z^{k-1}+\cdots+c_{1} z+c_{0} \quad$ and $\quad p_{\ell}(z)=c_{\ell} z^{\ell}+$ $c_{\ell-1} z^{\ell-1}+\cdots+c_{1} z+c_{0}$ are polynomials with complex coefficients.

Let $\left\{z_{1}, z_{2}, \ldots, z_{k}\right\}$ be the set of zeros of $p_{k}(z)=0$ and $\left\{z_{1}^{*}, z_{2}^{*}, \ldots, z_{\ell}^{*}\right\}$ be the set of zeros of $p_{\ell}(z)=0$ (recall that these zeros are complex numbers). Then $\mathcal{S}_{1}=\left\{z_{1} e_{+}, \ldots, z_{k} e_{+}\right\}, \mathcal{S}_{2}=\left\{z_{1}^{*} e_{-}, \ldots, z_{\ell}^{*} e_{-}\right\}$, where the set of zeros $\mathcal{S}=\mathcal{S}_{1} \oplus \mathcal{S}_{2}$ of equation (1) consists of $k \times \ell$ elements $z_{s, t}=z_{s} e_{+}+z_{t}^{*} e_{-}$; $s=1,2, \ldots, k$ and $t=1,2, \ldots \ell$.

Example 1 Let us consider the following equation

$$
2 x^{2}+(-1-i+j-e) x+(1+i-j-e)=0 .
$$

This equation can be reduced to

$$
\left\{\begin{array}{r}
\left(x^{2}-(i+1) x+i\right) e_{+}=0 \\
\left(x^{2}+1\right) e_{-}=0 .
\end{array}\right.
$$

Thus, it can be easily verified that

$$
\mathcal{S}_{1}=\left\{z_{1}=\frac{1+e}{2}, \quad z_{2}=\frac{i-j}{2}\right\}
$$

and

$$
\mathcal{S}_{2}=\left\{z_{1}^{*}=\frac{i+j}{2}, \quad z_{2}^{*}=\frac{-i-j}{2}\right\} .
$$


So, the four roots of equation (4) are given by

$$
\left\{\begin{array}{l}
x_{1}=z_{1}+z_{1}^{*}=\frac{1+i+j+e}{2}, \\
x_{2}=z_{2}+z_{1}^{*}=i \\
x_{3}=z_{1}+z_{2}^{*}=\frac{1-i-j+e}{2}, \\
x_{4}=z_{2}+z_{2}^{*}=-j .
\end{array}\right.
$$

Example 2 Now, let us consider the simple equation

$$
x^{3}-1=0 \text {. }
$$

This equation can be reduced to

$$
\left\{\begin{array}{l}
\left(x^{3}-1\right) e_{+}=0 \\
\left(x^{3}-1\right) e_{-}=0
\end{array}\right.
$$

Thus, it can be easily verified that

$$
\mathcal{S}_{1}=\left\{z_{1}=\frac{1+e}{2}, \quad z_{2}=-\frac{1}{4}+\frac{\sqrt{3}}{4} i-\frac{\sqrt{3}}{4} j-\frac{1}{4} e, \quad z_{3}=-\frac{1}{4}-\frac{\sqrt{3}}{4} i+\frac{\sqrt{3}}{4} j-\frac{1}{4} e\right\}
$$

and

$$
\mathcal{S}_{2}=\left\{z_{1}^{*}=\frac{1-e}{2}, \quad z_{2}^{*}=-\frac{1}{4}+\frac{\sqrt{3}}{4} i+\frac{\sqrt{3}}{4} j+\frac{1}{4} e, \quad z_{3}^{*}=-\frac{1}{4}-\frac{\sqrt{3}}{4} i-\frac{\sqrt{3}}{4} j+\frac{1}{4} e\right\} .
$$

So, the nine roots of (7) are given by

$$
\left\{\begin{array}{l}
x_{1}=z_{1}+z_{1}^{*}=1 \\
x_{2}=z_{1}+z_{2}^{*}=\frac{1}{4}+\frac{\sqrt{3}}{4} i-\frac{\sqrt{3}}{4} j+\frac{3}{4} e \\
x_{3}=z_{1}+z_{3}^{*}=\frac{1}{4}-\frac{\sqrt{3}}{4} i+\frac{\sqrt{3}}{4} j+\frac{3}{4} e \\
x_{4}=z_{2}+z_{1}^{*}=\frac{1}{4}+\frac{\sqrt{3}}{4} i-\frac{\sqrt{3}}{4} j-\frac{3}{4} e \\
x_{5}=z_{2}+z_{2}^{*}=-\frac{1}{2}+\frac{\sqrt{3}}{2} i \\
x_{6}=z_{2}+z_{3}^{*}=-\frac{1}{2}-\frac{\sqrt{3}}{2} j \\
x_{7}=z_{3}+z_{1}^{*}=\frac{1}{4}-\frac{\sqrt{3}}{4} i+\frac{\sqrt{3}}{4} j-\frac{3}{4} e \\
x_{8}=z_{3}+z_{2}^{*}=-\frac{1}{2}+\frac{\sqrt{3}}{2} j \\
x_{9}=z_{3}+z_{3}^{*}=-\frac{1}{2}-\frac{\sqrt{3}}{2} i .
\end{array}\right.
$$


It is easily verified that all roots $x_{i}, i=1,2, \ldots, 9$ lie on the unit sphere with center in 0 .

We should notice that equation (7) has a non-unique factorization. For instance, $x^{3}-1=(x-1)\left(x-x_{5}\right)\left(x-x_{9}\right)=(x-1)\left(x-x_{8}\right)\left(x-x_{6}\right)=\cdots$.

(ii) All coefficients of the first equation of system (2) are equal to zero, i.e., $a_{i}^{+}=0$; $i=0,1, \ldots, n$, but not all coefficients of the second equation of system (2) are equal to zero, otherwise all coefficients of polynomial $p_{n}(w)$ are zero. So, $\mathcal{S}_{1}=I\left(e_{+}\right)$and let the set of zeros of the second equation be $\mathcal{S}_{2}=$ $\left\{z_{1}^{*} e_{-}, \ldots, z_{\ell}^{*} e_{-}\right\}$. Therefore, $\mathcal{S}=\left\{a_{t} \mid a_{t}=z_{t}^{*} e_{-}+b ; b \in I\left(e_{+}\right) ; t=1,2, \ldots, \ell\right\}$.

(iii) All coefficients of the second equation of system (2) are equal to zero, i.e., $a_{i}^{-}=0 ; i=0,1, \ldots, n$, nonetheless not all coefficients of the first equation of system (2) are equal to zero. So, $\mathcal{S}_{2}=I\left(e_{-}\right)$and let the set of zeros of the first equation be $\mathcal{S}_{1}=\left\{z_{1} e_{+}, \ldots, z_{k} e_{+}\right\}$. Hence, $\mathcal{S}=\left\{a_{s} \mid a_{s}=z_{s} e_{+}+b ; b \in I\left(e_{-}\right)\right.$; $s=1,2, \ldots, k\}$.

Example 3 Let us consider the fourth-order equation

$$
(1+e) x^{4}-16 e-16=0 .
$$

This equation can be reduced to

$$
\left\{\begin{array}{r}
\left(x^{4}-16\right) e_{+}=0, \\
0 e_{-}=0 .
\end{array}\right.
$$

Thus, it can be easily verified that

$$
\mathcal{S}_{1}=\left\{z_{1}=1+e, z_{2}=-1-e, z_{3}=i-j, z_{4}=-i+j\right\}
$$

and $\mathcal{S}_{2}=I\left(e_{-}\right)$. So, the four roots of $(10)$ are given by

$$
\left\{\begin{array}{l}
x_{1}=1+e+z, \\
x_{2}=-1-e+z, \\
x_{3}=i-j+z, \\
x_{4}=-i+j+z,
\end{array}\right.
$$

where $z \in I\left(e_{-}\right)$.

(iv) The polynomial in equation (1) is of the form $a_{n}^{+} w_{+}^{n}+a_{n-1}^{+} w_{+}^{n-1}+\cdots+$ $a_{1}^{+} w_{+}+a_{0}=0$, where $a_{0}=a_{0}^{+}+a_{0}^{-}$and $a_{0}^{-} \neq 0$ or $a_{n}^{-} w_{-}^{n}+a_{n-1}^{-} w_{-}^{n-1}+\cdots+$ $a_{1}^{-} w_{-}+a_{0}=0$, and $a_{0}^{+} \neq 0$. In these cases equation (1) has no solution.

Example 4 Consider the equation

$$
(1+e) x^{2}+2=0 .
$$

This equation can be reduced to

$$
\left\{\begin{array}{r}
\left(x^{2}+1\right) e_{+}=0, \\
e_{-}=0,
\end{array}\right.
$$

which have no roots. 


\section{Conclusions}

Bicomplex algebra has been the object of much research of mathematicians and physicists in recent years. This interest is driven by possible applications of this algebra in physics and mathematics [3]. However, in most of these works the focus is on understanding the main properties of holomorphic functions defined on the space of bicomplex algebra, e.g., the bicomplex generalization of Cauchy's theorem and related integral formulae, and so on [1-4]. In this article, we have presented a method to find the roots or zeros of bicomplex polynomial equations, which may arise in problems of finding eigenvalues of operators over bicomplex algebra, roots of bicomoplex functions, and many others. Our analysis has revealed the interesting fact of a non-unique factorization of bicomplex polynomials.

We hope that our results will be useful for further investigations regarding these interesting numbers.

\section{Acknowledgements}

The authors would like to thank ITESM, Campus Monterrey, through the Research Chair in Telecommunications for the support provided in the development of this work.

\section{References}

[1] Segre, C., 1892, Le rappresentazioni reali delle forme complesse a gli enti iperalgebrici. Mathematische Annalen, 40, 413-467.

[2] Rochon, D. and Shapiro, M., 2004, On algebraic properties of bicomplex and hyperbolic numbers. Analele Universitatii din Oradea. Fascicola Matematica, 11, 1-28.

[3] Turbin, A.F., 1999, Hypercomplex analysis in Lyush's algebra. Problems of analytical mechanics and its applications, Proceedings of the Mathematical Institute of the NAS of Ukraine, 26, 387-406.

[4] Rönn, S., 2001, Bicomplex algebra and function theory. arXiv:math.CV/0101200v1, Vol. 1, 1-71.

[5] Pogorui, A.A. and Shapiro, M., 2004, On the structure of the set of the zeros of quaternionic polynomials. Complex Variables, 49(6), 379-389. 